\title{
The double-layer of penetrable ions: An alternative route to charge reversal
}

Cite as: J. Chem. Phys. 138, 174901 (2013); https://doi.org/10.1063/1.4802994

Submitted: 26 March 2013 . Accepted: 12 April 2013 . Published Online: 01 May 2013

Derek Frydel, and Yan Levin

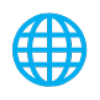

View Online

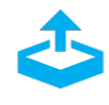

Export Citation

\section{ARTICLES YOU MAY BE INTERESTED IN}

The double-layer structure of overscreened surfaces by smeared-out ions

The Journal of Chemical Physics 145, 184703 (2016); https://doi.org/10.1063/1.4967257

Polarizable Poisson-Boltzmann equation: The study of polarizability effects on the structure of a double layer

The Journal of Chemical Physics 134, 234704 (2011); https://doi.org/10.1063/1.3598476

Bjerrum pairs in ionic solutions: A Poisson-Boltzmann approach

The Journal of Chemical Physics 146, 194904 (2017); https://doi.org/10.1063/1.4982885

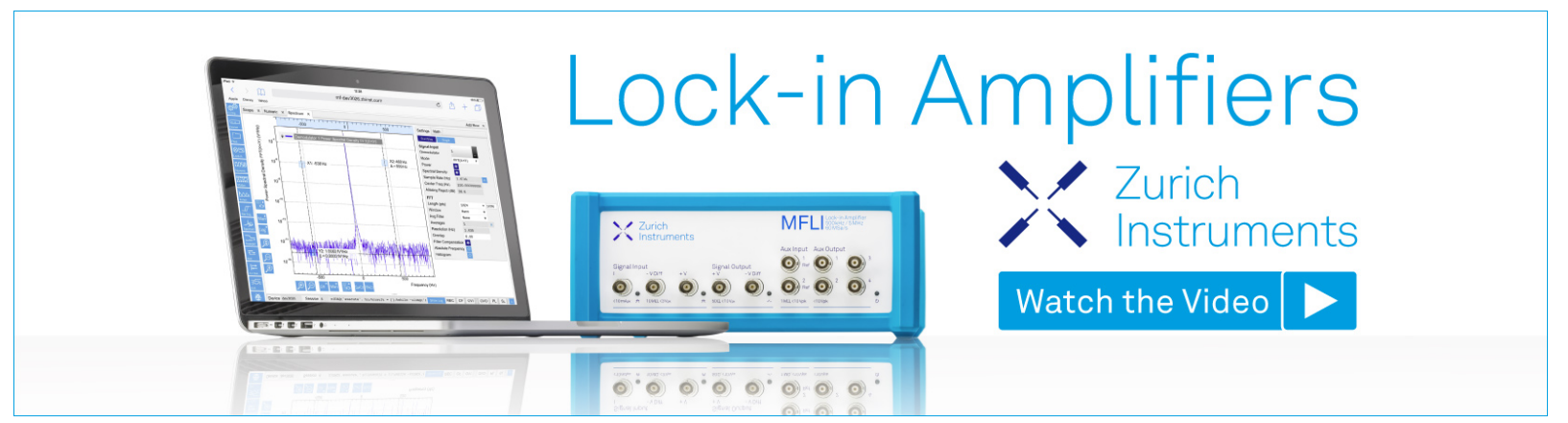

J. Chem. Phys. 138, 174901 (2013); https://doi.org/10.1063/1.4802994 


\title{
The double-layer of penetrable ions: An alternative route to charge reversal
}

\author{
Derek Frydel and Yan Levin \\ Institute of Physics, The Federal University of Rio Grande do Sul, PO Box 15051, \\ 91501-970 Porto Alegre, RS, Brazil
}

(Received 26 March 2013; accepted 12 April 2013; published online 1 May 2013)

\begin{abstract}
We investigate a double-layer of penetrable ions near a charged wall. We find a new mechanism for charge reversal that occurs in the weak-coupling regime and, accordingly, the system is suitable for the mean-field analysis. The penetrability is achieved by smearing-out the ionic charge inside a sphere, so there is no need to introduce non-electrostatic forces and the system in the low coupling limit can be described by a modified version of the Poisson-Boltzmann equation. The predictions of the theory are compared with the Monte Carlo simulations. (C) 2013 AIP Publishing LLC. [http://dx.doi.org/10.1063/1.4802994]
\end{abstract}

\section{INTRODUCTION}

Overcharging is a surprising phenomenon in which counterions adsorbed to a surface exceed the number of fixed surface charges. ${ }^{1}$ As a consequence, coions are drawn from the bulk toward the overcharged surface leading to a formation of a "triple-layer." Because the underlying mechanism of overcharging relies on electrostatic correlations, ${ }^{2}$ it has become synonymous with the strong-coupling limit. Indeed, a mean-field treatment which accurately captures the weakcoupling limit, cannot describe overcharging. (However, the mean-field in combination with the excluded volume interactions can induce overcharging if the bulk volume fraction of an electrolyte is sufficiently large to generate a depletion force that pushes particles against a surface. This effect is seen in uncharged systems and persists for weakly charged surface charges. ${ }^{3-6}$ )

In the strong-coupling limit, overcharging is the result of increased structuring within the layer of counterions. The electrostatic correlations between the condensed counterions lead to formation of correlation "holes" within the layer of condensed ions which can attract excess of counterions from the bulk. The value of the coupling constant $\Gamma=Z^{3} \lambda_{B} / \lambda_{\mathrm{GC}}$, which is the ratio between the Bjerrum and Gouy-Chapman length ( $Z$ is the valency), estimates the extent of correlation effects. In the limit $\Gamma \rightarrow \infty$, the counterions are said to freeze into a $2 \mathrm{D}$ Wigner crystal. ${ }^{8}$ At large, but finite $\Gamma$, the local structure of an ionic fluid remains Wigner-like. ${ }^{7-10}$ The above mechanism is specific to Coulomb interactions that diverge as $r \rightarrow 0$ and, therefore, exhibit the excluded volume effects. ${ }^{9}{ }^{11}$ If, however, the divergence in the pair interaction is removed (the pair potential is bounded as $r \rightarrow 0$ ), particles can interpenetrate and the usual excluded volume interactions underlying the crystal formation are eliminated (at sufficiently high temperature and/or density). For some family of bounded potentials, particles can form stacks where two or more particles occupy the same position and act as an effective single particle. This is possible only if a pair potential is sufficiently flat around $r=0 .{ }^{12}$ One example is the penetrable sphere model where the pair potential is the step function. ${ }^{13-16}$ The stacking formations stabilize the liq- uid phase, ${ }^{14}$ since doublets, triplets, etc., effectively decrease the number of particles. The presence of stacked formations is signaled as a positive peak in the pair correlation function at $r \rightarrow 0 .{ }^{14}$ Extrapolating these ideas to ions, which in addition to penetrable cores have long-range Coulomb interactions, we ask what influence penetrability has on the structure of a double-layer. Can the restructuring invoked by the penetrability lead to overcharging in the weak-coupling limit?

In the present work, the divergence in the Coulomb potential is removed by smearing-out the central charge of an ion over a finite region. The penetrating core, then, depends on the weight function used to smear out the charge. This procedure does not require going beyond the framework of electrostatics and the weak-coupling limit can be described by the modified version of the Poisson-Boltzmann (PB) equation.

Bulk properties and phase diagram of penetrable ions have been investigated in Refs. 17-19. The main feature is the formation of the Bjerrum pairs of two opposite ions that function as polarizable particles. The formation of these pairs leads to an insulator-conductor transition, ${ }^{17}$ which does not exist in systems of hard-core ions in three dimensions. ${ }^{20}$ Thus, penetrability dramatically affects the phase transition and the topology of the phase diagram.

The model of penetrable particles is not only of theoretical interest. Various macroparticles can exhibit interpenetration. Marquest and Witten ${ }^{13}$ suggested a penetrable sphere model for micelles. Polymer coils and dendrimers in good solvent can be represented by a Gaussian core model. ${ }^{21,22}$ If in addition these macroparticles are charged, as is often the case for real systems, ${ }^{23}$ then the model of penetrable ions can be of genuine physical relevance. Recently, ionic microgels have been modeled as uniformly charged spheres ${ }^{24}$ allowing interpenetration at short- and Coulomb-interactions at longseparations.

\section{THE POISSON-BOLTZMANN EQUATION FOR SMEARED-OUT CHARGES}

The charge $q_{i}$ of an ion $i$ is smeared out over the finite region according to the weight function $\omega_{i}\left(\mathbf{r}-\mathbf{r}_{i}\right)$ such 
that $\int d \mathbf{r} \omega_{i}\left(\mathbf{r}-\mathbf{r}_{i}\right)=q_{i}$. The charge density operator for $N$ smeared-out ions is

$$
\hat{\rho}_{c}(\mathbf{r})=\sum_{i=1}^{N} \int d \mathbf{r}^{\prime} \delta\left(\mathbf{r}^{\prime}-\mathbf{r}_{i}\right) \omega_{i}\left(\mathbf{r}^{\prime}-\mathbf{r}\right) .
$$

In this work we consider a symmetric electrolyte, $q_{+}=-q_{-}$. The averaged charge density for this system is

$$
\rho_{c}(\mathbf{r})=\int d \mathbf{r}^{\prime}\left[\rho_{+}\left(\mathbf{r}^{\prime}\right) \omega_{+}\left(\mathbf{r}^{\prime}-\mathbf{r}\right)+\rho_{-}\left(\mathbf{r}^{\prime}\right) \omega_{-}\left(\mathbf{r}^{\prime}-\mathbf{r}\right)\right],
$$

where $\rho_{+}$and $\rho_{-}$denote the number densities of anions and cations, respectively. The Poisson equation is

$$
\epsilon \nabla^{2} \psi=-\int d \mathbf{r}^{\prime}\left[\rho_{+}\left(\mathbf{r}^{\prime}\right) \omega_{+}\left(\mathbf{r}^{\prime}-\mathbf{r}\right)+\rho_{-}\left(\mathbf{r}^{\prime}\right) \omega_{-}\left(\mathbf{r}^{\prime}-\mathbf{r}\right)\right],
$$

where $\epsilon$ is the background dielectric constant. To obtain a closed equation, we need an expression for $\rho_{-}$and $\rho_{+}$in terms of the mean electrostatic potential $\psi$. For point charges, this leads to

$$
\rho_{\alpha}(\mathbf{r})=c_{s} e^{-\beta q_{\alpha} \psi(\mathbf{r})},
$$

where the subscript $\alpha$ is either + or - , and $c_{s}$ is the bulk salt concentration. The number density depends locally on an electrostatic potential. However, if charge is smeared around the ion center at $\mathbf{r}$, the entire distribution $\omega\left(\mathbf{r}^{\prime}-\mathbf{r}\right)$ interacts with the mean electrostatic potential,

$$
\psi_{\alpha}(\mathbf{r})=\int d \mathbf{r}^{\prime} \psi\left(\mathbf{r}^{\prime}\right) \omega_{\alpha}\left(\mathbf{r}^{\prime}-\mathbf{r}\right),
$$

and the number density becomes

$$
\rho_{\alpha}=c_{s} e^{-\beta \int d \mathbf{r}^{\prime} \psi\left(\mathbf{r}^{\prime}\right) \omega_{\alpha}\left(\mathbf{r}^{\prime}-\mathbf{r}\right)} .
$$

We may now write down the mean-field equation for the electrostatic potential produced by smeared-out ions,

$$
\begin{aligned}
-\epsilon \nabla^{2} \psi= & c_{s} \int d \mathbf{r}^{\prime} \omega_{+}\left(\mathbf{r}^{\prime}-\mathbf{r}\right) e^{-\beta \int d \mathbf{r}^{\prime \prime} \psi\left(\mathbf{r}^{\prime \prime}\right) \omega_{+}\left(\mathbf{r}^{\prime \prime}-\mathbf{r}^{\prime}\right)} \\
& +c_{s} \int d \mathbf{r}^{\prime} \omega_{-}\left(\mathbf{r}^{\prime}-\mathbf{r}\right) e^{-\beta \int d \mathbf{r}^{\prime \prime} \psi\left(\mathbf{r}^{\prime \prime}\right) \omega_{-}\left(\mathbf{r}^{\prime \prime}-\mathbf{r}^{\prime}\right)}
\end{aligned}
$$

We refer to this modified Poisson-Boltzmann equation as the Finite-Spread PB equation (FSPB). The FSPB equation complements the already quite sizable set of modified PB equations: the $\mathrm{PB}$ that incorporates the excluded volume interactions, ${ }^{25}$ the dipolar interactions, ${ }^{26}$ the nonlinear solvent contributions, ${ }^{27}$ and the polarizability of ions. ${ }^{28,29}$ The idea of finitely spread-out ions was considered in Ref. 30 to study the self energy contributions beyond the mean-field. Mathematically, the FSPB equation has the structure of a convoluted equation. Each ion is convoluted according to the weight function, which determines the composition of a single ion.

Equation (7) can also be obtained by minimizing the grand potential,

$$
\begin{aligned}
\Omega\left[\left\{\rho_{\alpha}\right\}\right]= & \frac{1}{2} \int d \mathbf{r} \int d \mathbf{r}^{\prime} \frac{\rho_{c}(\mathbf{r}) \rho_{c}\left(\mathbf{r}^{\prime}\right)}{4 \pi \epsilon\left|\mathbf{r}^{\prime}-\mathbf{r}\right|} \\
& +k_{B} T \sum_{\alpha} \int d \mathbf{r} \rho_{\alpha}(\mathbf{r})\left[\log \rho_{\alpha} \Lambda^{3}-1\right] \\
& -\sum_{\alpha} \mu \int d \mathbf{r} \rho_{\alpha}(\mathbf{r})
\end{aligned}
$$

where $\Lambda$ is the thermal de Broglie wavelength and $\mu=\mu_{+}$ $=\mu_{-}$is the chemical potential. The three contributions are the electrostatic energy, entropy, and we allow the number density to fluctuate through the contact with a reservoir. The minimization $\frac{\delta \Omega}{\delta \rho_{\alpha}}=0$ recovers Eq. (6) and the application of the Poisson equation yields the FSPB model.

\section{VARIOUS DISTRIBUTIONS $\omega(r)$}

The concept of charge smearing is not novel to this work, but it has been evoked many times in the past both as a physical representation and a mathematical construct. The best known example (and to our knowledge the earliest) of mathematical construct is the calculation of Ewald summation to treat periodic charges, ${ }^{31}$ today the most practiced method to account for contributions due to periodic boundary conditions of a simulated system, ${ }^{32}$ where by spreading the charge one achieves separation of interactions into the short- and longrange counterpart. Another instance of mathematical convenience is the Onsager smearing optimization to obtain the variational free energy of the strongly correlated one component plasma. ${ }^{33}$ The idea of charges which at short separations exhibits soft repulsion and at long separations the Coulomb interaction appeared in Ref. 34 to represent semi-classical hydrogen plasma at high temperature and density, yet no smearing procedure was used to construct this potential. The actual smearing procedure to represent physical particles was used to model electrons set in cyclotron motion by a uniform magnetic field. ${ }^{35,36}$ In soft matter, the smearing out procedure was used to represent microgels in Ref. 37 , and recently to represent charged polymers. ${ }^{17-19}$

We consider a few simple, spherically symmetric distributions $\omega(r)$ and the interactions that result from these distributions. A spherically symmetric charge distribution generates the following electrostatic potential:

$$
\psi(r)=\frac{1}{\epsilon}\left[\frac{1}{r} \int_{0}^{r} d s s^{2} \omega(s)+\int_{r}^{\infty} d s s \omega(s)\right] .
$$

To derive the above formula we applied the Gauss law to the Poisson equation, and afterwards we integrated the resulting electrostatic field (the integration by parts was evoked). If the distribution is uniform inside a spherical cavity,

$$
\omega\left(\mathbf{r}^{\prime}-\mathbf{r}\right)=\frac{3 Q}{4 \pi R^{3}} \theta\left(R-\left|\mathbf{r}^{\prime}-\mathbf{r}\right|\right),
$$

where $\theta$ is the Heaviside function and $\int d \mathbf{r}^{\prime} \omega\left(\mathbf{r}^{\prime}-\mathbf{r}\right)=Q$, then the potential inside a sphere is

$$
\psi(r \leq R)=\frac{Q}{4 \pi \epsilon}\left[\frac{3 R^{2}-r^{2}}{2 R^{3}}\right] .
$$

Outside the sphere, the Coulomb potential is recovered. The interaction between two ions with this charge distribution is $^{33,37}$

$$
U(r \leq 2 R)=\frac{Q^{2}}{4 \pi \epsilon}\left[\frac{192 R^{5}-80 R^{3} r^{2}+30 R^{2} r^{3}-r^{5}}{160 R^{6}}\right] .
$$


For non-overlapping separations, ions behave like point charges. Next, we consider the distribution

$$
\omega\left(\mathbf{r}^{\prime}-\mathbf{r}\right)=\frac{Q}{4 \pi R^{2}} \delta\left(R-\left|\mathbf{r}^{\prime}-\mathbf{r}\right|\right),
$$

where a charge is smeared-out over a spherical shell. Inside a sphere, the electrostatic potential is constant,

$$
\psi(r \leq R)=\frac{Q}{4 \pi \epsilon} \frac{1}{R} .
$$

The interaction between two of these distributions for overlapping separations is

$$
U(r \leq 2 R)=\frac{Q^{2}}{4 \pi \epsilon}\left[\frac{4 R-r}{4 R^{2}}\right] .
$$

A sphere and a shell distribution interact via

$$
U(r \leq 2 R)=\frac{Q^{2}}{4 \pi \epsilon}\left[\frac{16 R^{3}-4 R r^{2}+r^{3}}{16 R^{4}}\right] .
$$

Finally, we consider a Gaussian distribution, ${ }^{17-19,31}$

$$
\omega\left(\mathbf{r}^{\prime}-\mathbf{r}\right)=\frac{Q^{2}}{(2 \pi)^{3 / 2} R^{3}} e^{-r^{2} / 2 R^{2}},
$$

which leads to the following pair interaction:

$$
U(r)=\frac{Q^{2}}{4 \pi \epsilon} \frac{\operatorname{erf}(r / 2 R)}{r} .
$$

\section{NUMERICAL RESULTS FOR THE FSPB EQUATION}

We primarily focus on the distribution

$$
\omega_{ \pm}\left(\left|\mathbf{r}-\mathbf{r}_{i}\right|\right)= \pm e \theta\left(R-\left|\mathbf{r}-\mathbf{r}_{i}\right|\right) / \nu .
$$

where $v=4 \pi R^{3} / 3$ is the ionic volume. The FSPB equation in reduced units reads

$$
\nabla^{2} \phi=\kappa^{2} v^{-1} \int d \mathbf{r}^{\prime} \theta\left(R-\left|\mathbf{r}^{\prime}-\mathbf{r}\right|\right) \sinh \left(\bar{\phi}_{\theta}\right),
$$

where

$$
\bar{\phi}_{\theta}(\mathbf{r})=\frac{1}{v} \int d \mathbf{r}^{\prime} \phi\left(\mathbf{r}^{\prime}\right) \theta\left(R-\left|\mathbf{r}^{\prime}-\mathbf{r}\right|\right)
$$

$\phi=\beta e \psi, \kappa^{-1}=1 / \sqrt{8 \pi \lambda_{B} c_{s}}$ is the screening length, and $\lambda_{B}$ $=\beta e^{2} / 4 \pi \epsilon$ is the Bjerrum length. The number density of each species is

$$
\rho_{ \pm}=c_{S} e^{\mp \bar{\phi}_{\theta}}
$$

All the ions occupy the half-space $x>0$. The charged hard wall at $x=-R$ determines boundary conditions,

$$
\frac{\partial \phi}{\partial x}=-4 \pi \lambda_{B} \sigma_{c},
$$

where $\sigma_{c}$ is the surface charge. Note that the number density is limited to the region $x>0$, but the charge density, because of the distribution $\omega(r)$, reaches all the way to
$x=-R$, the location of the charged wall. Consequently, we assume the (hard-sphere)-(hard-wall) potential between an ion and the wall. Subsequent figures show data points for $x>0$, the region available to ion centers.

In the dilute limit $\left(c_{s} \rightarrow 0\right)$, the solution of the PB equation for point ions in the weak-coupling limit yields an algebraic density profile,

$$
\rho(x)=\frac{2 \pi \lambda_{B} \sigma_{c}^{2}}{\left(1+x \lambda_{\mathrm{GC}}^{-1}\right)^{2}} .
$$

In the strong-coupling limit the electrostatic correlations modify the functional form of the distribution, ${ }^{38}$

$$
\rho(x)=2 \pi \lambda_{B} \sigma_{c}^{2} \exp \left(-x \lambda_{\mathrm{GC}}^{-1}\right),
$$

where $\lambda_{\mathrm{GC}}=\left(2 \pi \lambda_{B} \sigma_{c}\right)^{-1}$ is the Gouy-Chapman length. The barometric-like distribution in the transverse direction is a consequence of high degree of ordering in the lateral direction reminiscent of the Wigner crystal. ${ }^{8,10}$

Although the FSPB equation is purely mean-field, we find similar modification of the density profile, but this time the relevant parameter is $R$. The limit $R \rightarrow 0$ corresponds to the ionic distribution given by Eq. (23), and the limit $R \rightarrow \infty$ to the one given by Eq. (24), see Fig. 1. In the limit $R \rightarrow \infty$, see Eq. (12), we recover an ideal gas in a uniform gravitational field.

Figure 2 shows the counterion profiles for an electrolyte solution. This introduces a length scale $\kappa^{-1}$. Counterions from the bulk can now overcharge the surface. The counterion profile beyond $x=0.5 \mathrm{~nm}$ dips below the bulk value, which indicates an overcharged surface. Figure 3 shows how the coion density rises above the bulk value, another signature of charge reversal. Finally, Fig. 4 shows the non-monotonic electrostatic potential which goes to negative values and has a minimum around $x=0.6 \mathrm{~nm}$, at which point the electrostatic field vanishes and, farther on, it changes sign.

Under what conditions penetrability of ions leads to charge reversal? For penetration to take place, either the thermal fluctuations must exceed the interaction energy of the overlapped particles, $\beta U(r=0) \lesssim 1$, or the counterion

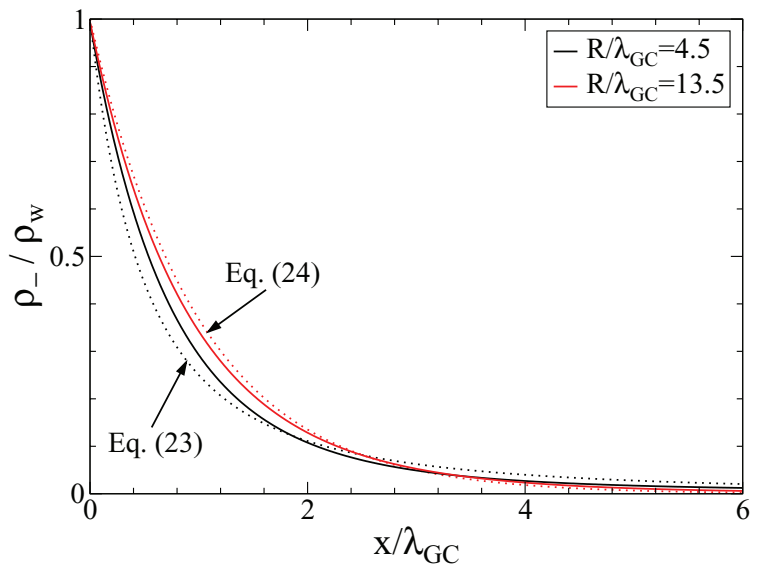

FIG. 1. The counterion density profile in the dilute limit (no coions). The dotted lines demarcate the limiting behaviors in Eqs. (23) and (24). The wall charge is positive and the counterions have negative charge. As $R$ increases, the profile takes the exponential shape. 


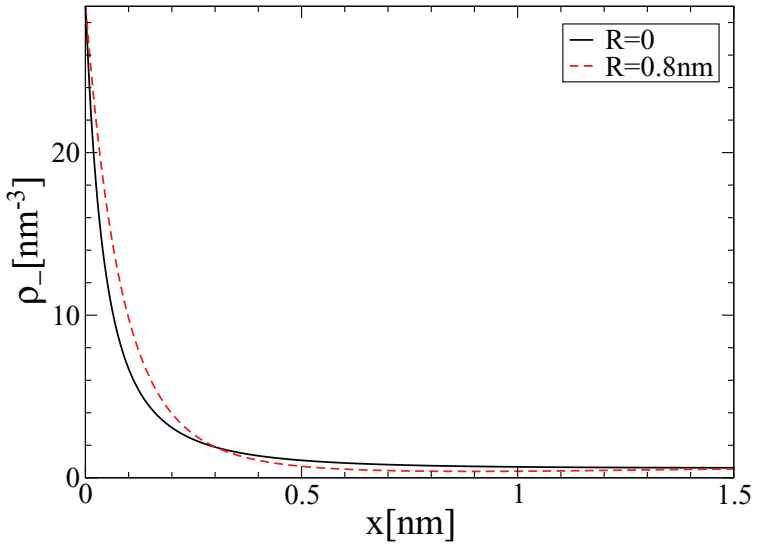

FIG. 2. The counterion density profile near a charged wall. $R=0$ corresponds to point ions. The relevant lengths are: $\kappa^{-1}=0.3 \mathrm{~nm}, \lambda_{B}=0.72 \mathrm{~nm}$, and $\lambda_{\mathrm{GC}}=0.09 \mathrm{~nm}$.

concentration at the wall must be sufficiently large to involve numerous overlaps, such that the effective $2 \mathrm{D}$ density within the counterion layer is $\rho_{2 D} R^{2} \gtrsim 1$. For the distribution in Eq. (19), the first requirement translates into $R / \lambda_{B} \gtrsim 1.2$, and, assuming $\rho_{2 D} \approx \sigma_{c}$, the second one becomes $\sigma_{c} R^{2} \gtrsim 1$. In Fig. $2, R / \lambda_{B} \approx 1.1$ and $R^{2} \sigma_{c} \approx 1.6$, and so both criteria are met.

As a parenthetical note, we point out that the PB and the FSPB models satisfy the same contact value relation,

$$
k_{B} T \rho_{w}=P_{\text {id }}+\frac{\sigma_{c}^{2}}{2 \epsilon},
$$

where $P_{\text {id }}=k_{B} T \rho_{b}$ is the ideal gas pressure in the bulk, $\rho_{b}$ and $\rho_{w}$ are the total densities in the bulk and at the contact with a wall, respectively.

\section{A. Tuning of the short-range interactions}

The sole constraint that $\omega\left(\mathbf{r}^{\prime}-\mathbf{r}\right)$ needs to satisfy is that its integral recovers the charge of an ion. This leaves sufficient room to manipulate the short-range interactions. As an

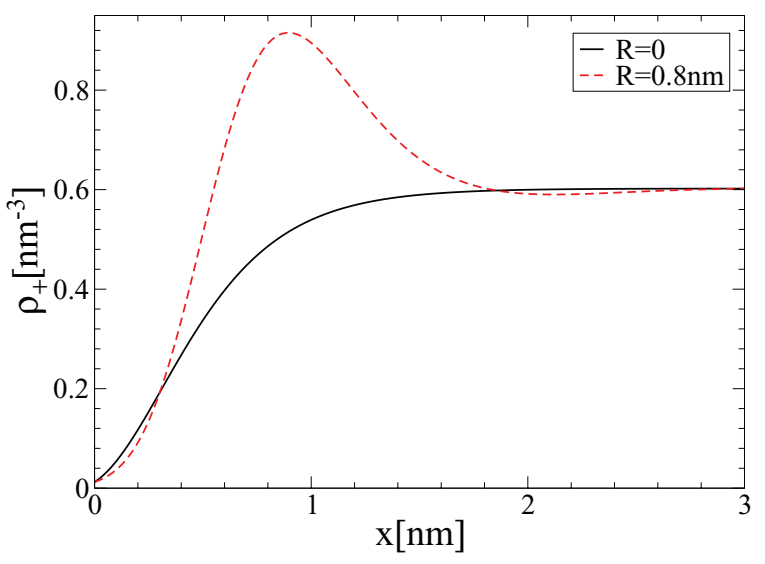

FIG. 3. The coion distribution at a charged wall corresponding to the system in Fig. 2. The coions are in excess to neutralize the inverted charge. Note that the density profile exhibits oscillations.

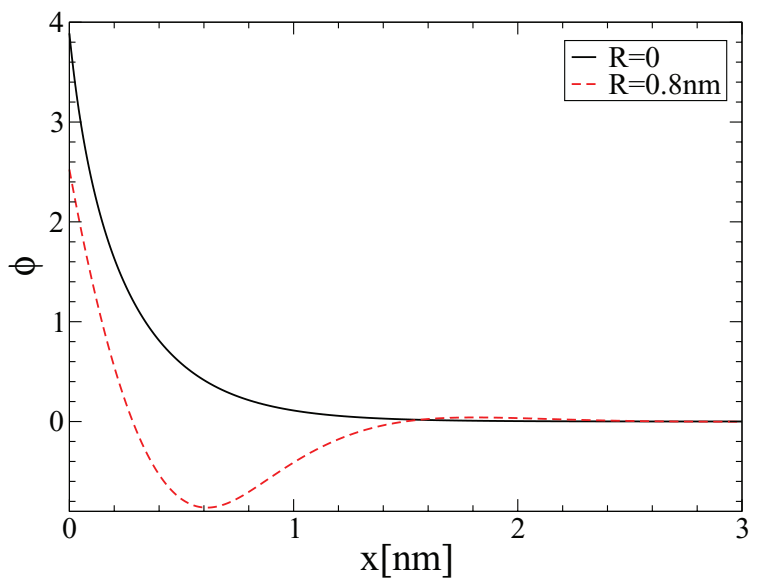

FIG. 4. Electrostatic potential near a charged wall corresponding to the system in Fig. 2. The minimum corresponds to a point of vanishing electrostatic field.

example, we can take a mixed distribution,

$$
\begin{aligned}
\omega_{ \pm}\left(\left|\mathbf{r}-\mathbf{r}_{i}\right|\right)= & \pm e\left[(\mathcal{Z}+1) \theta\left(R-\left|\mathbf{r}-\mathbf{r}_{i}\right|\right) / \nu\right. \\
& \left.-\mathcal{Z} \delta\left(R-\left|\mathbf{r}-\mathbf{r}_{i}\right|\right) / \gamma\right],
\end{aligned}
$$

comprised of a charged shell and sphere. $\gamma=4 \pi R^{2}$ is the surface area of a sphere, $\mathcal{Z}$ is the additional parameter, and $\mathcal{Z}=0$ corresponds to the distribution in Eq. (19). The number density is

$$
\rho_{ \pm}=c_{s} e^{\mp\left[(\mathcal{Z}+1) \bar{\phi}_{\theta}-\mathcal{Z} \bar{\phi}_{\delta}\right]},
$$

where

$$
\bar{\phi}_{\delta}(\mathbf{r})=\frac{1}{\gamma} \int d \mathbf{r}^{\prime} \phi\left(\mathbf{r}^{\prime}\right) \delta\left(R-\left|\mathbf{r}^{\prime}-\mathbf{r}\right|\right) .
$$

Figure 5 plots the pair potentials for different $\mathcal{Z}$. The parameter $\mathcal{Z}$ controls the strength of the repulsion and, by the same token, the strength of the excluded volume interactions. By way of example, Fig. 6 shows the density profile for $\mathcal{Z}=0$ and $\mathcal{Z}=4$, for $R=0.8 \mathrm{~nm}$. The excluded volume contributions for $\mathcal{Z}=4$ expel counterions from the first layer, which is the opposite of overcharging seen for $\mathcal{Z}=0$.

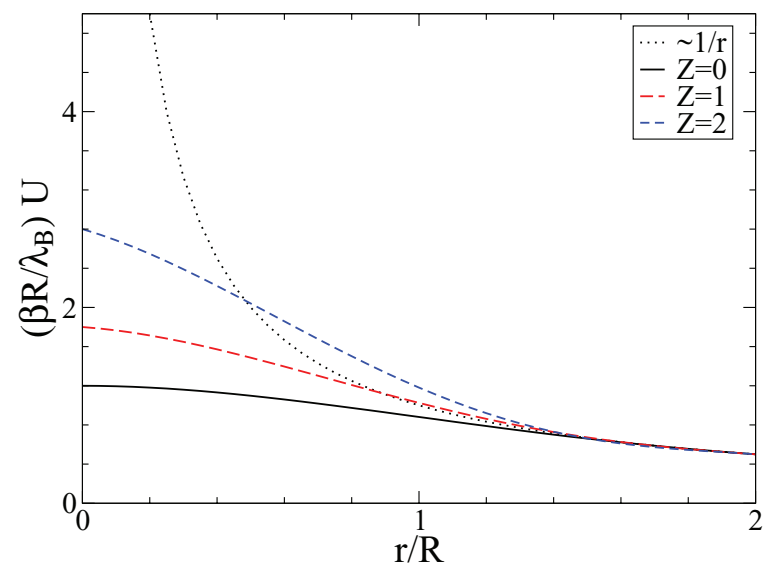

FIG. 5. Pair potential for two overlapping identical ions with charge distribution in Eq. (26). $\mathcal{Z}=0$ corresponds to the distribution in Eq. (19). 


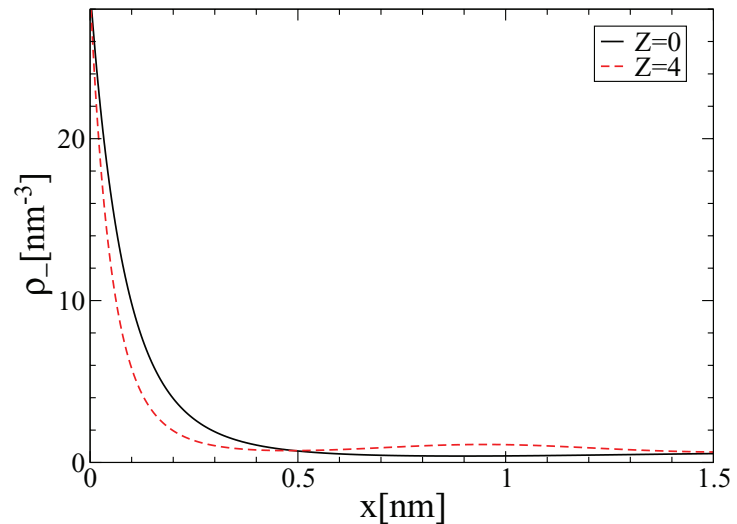

FIG. 6. The counterion density profile near a charged wall for the point ions and for the ions with the distribution in Eq. (26) for $R=0.8 \mathrm{~nm}$ and different $\mathcal{Z}$. The system parameters are those in Fig. 2.

With this example, we try to demonstrate possible extensions of the model based on charge spreading. For example, the repulsion controlled by the parameter $\mathcal{Z}$ could represent the interaction between polymers on account of the selfavoiding walk of polymer chains.

\section{COMPARISON WITH SIMULATION}

To test the mean-field approximation implicit in the FSPB equation, we carry out Monte Carlo (MC) simulations. The size of the simulation box is $L_{y}=L_{z}=8 \mathrm{~nm}$ and $L_{x}$ $=6 \mathrm{~nm}$. The periodic boundary conditions are in the lateral $(y, z)$-directions. The bounding plates at $x=0$ and $x=L_{x}$ have opposite charge. The box encloses $N=600$ ions, $N_{+}$ $=N_{-}=300$. To check for the finite size effects, we double the simulation size for some parameters. The standard Ewald summation is applied for contributions of periodic images. ${ }^{32}$ If particles overlap, we supplement the interaction energy with the term: $\beta \Delta U_{\text {tot }}(r<2 R)=\beta U\left(r_{i j}\right)-\frac{\lambda_{B}}{r_{i j}}$. (A physical picture is slightly modified when doing simulations. In simulations we tend to think of particles as point charges which at separations $r<2 R$ switch to the non-Coulomb interactions $U(r)$. Within the mean-field theory based on the Poisson equation, we tend to think of an ion as a charged ball. The two conceptions are, however, identical.)

Figure 7 compares counterion profiles obtained from the simulation and the FSPB equation. For $R=0.8 \mathrm{~nm}$ the agreement is virtually exact. For the smaller radius $R=0.1 \mathrm{~nm}$ we see the two results deviate: in the simulation, the correlations generate a more concentrated layer of counterions (but no overcharging). In Fig. 8, we plot the coion density profile for $R=0.8 \mathrm{~nm}$ to further confirm the accuracy of the meanfield in this regime.

What causes charge reversal for smeared-out ions? Do counterions merely penetrate or the pair potential is sufficiently flat in the overlap region and counterions collapse into stacked formations, so that the pair correlation function exhibits positive peak as $r \rightarrow 0$ ? In Fig. 9, we show the configuration snapshots for counterions adsorbed on a charged surface for different values of $R$. The difference in structure is

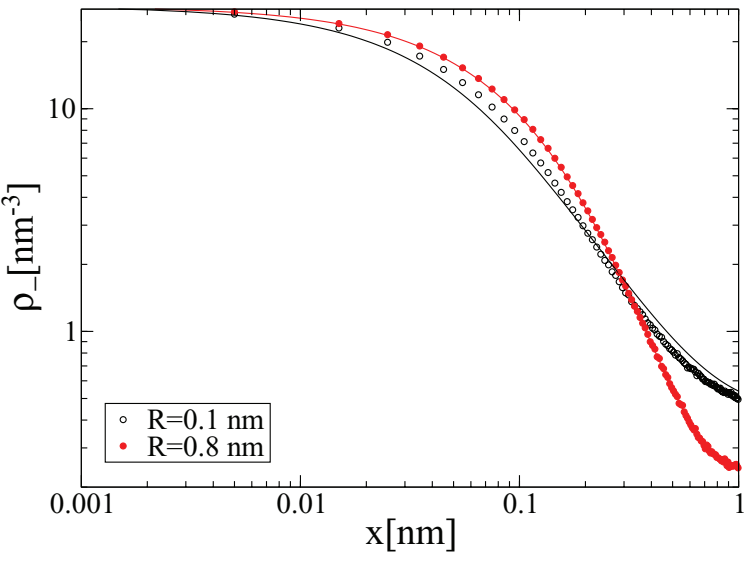

FIG. 7. Counterion density profiles. The system parameters are: $\lambda_{\mathrm{GC}}=0.09 \mathrm{~nm}$ and $\lambda_{B}=0.72 \mathrm{~nm}$. The symbols designate the simulation data points and the lines are the numerical results for the FSPB equation.

perceptible. Ionic penetration favors smaller separations between counterions. This gives the impression of string-like formations. As mentioned in the introduction, not all penetrable potentials can lead to stacked configurations, where two or more particles occupy "the same" space. For example, the Gaussian core model precludes, ${ }^{39,40}$ while the penetrable sphere model favors the stacked configurations. ${ }^{14,15}$ Stable stacked aggregates require that a pair potential be sufficiently flat. A more rigorous test involves the Fourier transform of the pair interaction. If $U(k)$ oscillates, and, therefore, involves negative values, then the stacking takes place under certain conditions. ${ }^{12}$ On the other hand, if $U(k)$ is non-negative, stacking does not occur under any conditions. This criterion is arrived at by considering the Ornstein-Zernike relation which, in the Fourier space, is

$$
h(k)=\frac{c(k)}{1-\rho c(k)},
$$

where $c(r)$ and $h(r)$ are the direct and pair correlation functions, respectively. In the mean-field approximation $c_{\mathrm{MF}}(r)$ $\approx-\beta U(r)$ [the exact definition is $c(r)=-\frac{\delta^{2} \beta F_{e x}}{\delta \rho(\mathbf{r}) \delta \rho\left(\mathbf{r}^{\prime}\right)}$, and in the

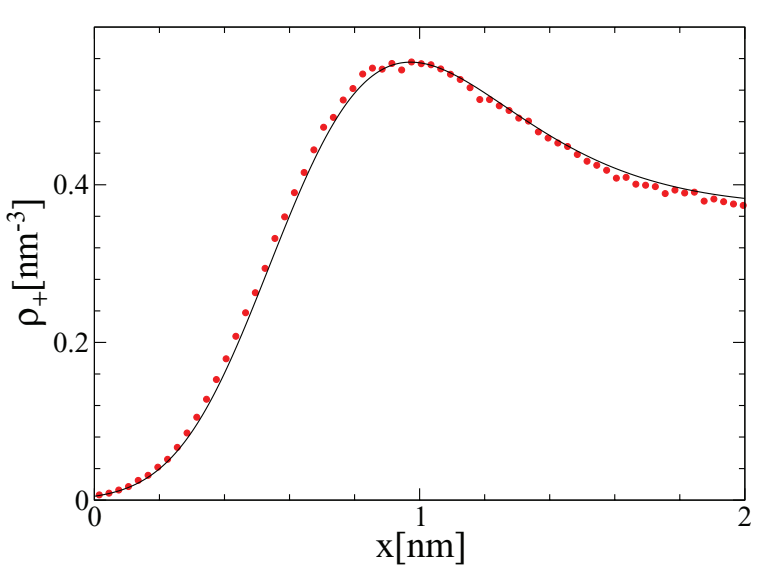

FIG. 8. The coion density profile for $R=0.8 \mathrm{~nm}$ obtained from the simulation (symbols) and the FSPB equation (solid line). The remaining parameters are as in Fig. 7. 

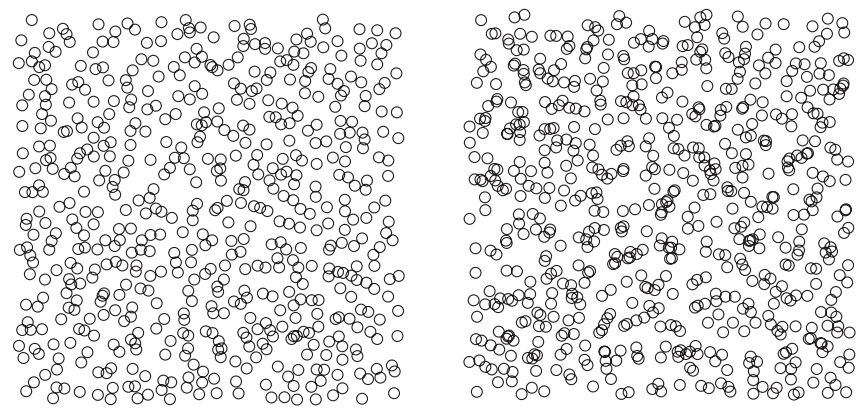

FIG. 9. Configuration snapshot of counterions adsorbed on the charged wall at $x<0.35 \mathrm{~nm}$. The diameter of particles is $\sigma=0.5 \mathrm{~nm}$ and is selected arbitrarily for visualization. The configuration on left is for $R / \lambda_{B}=0.14$ and the one on the right is for $R / \lambda_{B}=1.1$. The $2 \mathrm{D}$ densities are $\rho_{2 d}=2.34 \mathrm{~nm}^{-2}$ and $\rho_{2 d}=2.68 \mathrm{~nm}^{-2}$, respectively. For comparison, the surface charge density is $\sigma_{c}=2.50 \mathrm{~nm}^{-2}$. The system parameters are as in Fig. 7 .

mean-field $F_{e x}$ is the first term in Eq. (8)], so that

$$
h_{\mathrm{MF}}=-\frac{\beta U(k)}{1+\rho \beta U(k)} .
$$

If we take $h(r=0)>0$ to be the signature of stacking, and

$$
h_{\mathrm{MF}}(0)=-\frac{1}{2 \pi^{2}} \int_{0}^{\infty} d k \frac{\beta U(k) k^{2}}{1+\rho \beta U(k)},
$$

then it becomes immediately clear that a non-negative $U(k)$ always yields $h_{\mathrm{MF}}(0)<0$, and no stacking occurs. Only an oscillating $U(k)$ can yield $h_{\mathrm{MF}}>0$. In the present model particles are smeared-out charges,

$$
\begin{gathered}
\beta U(k)=\frac{4 \pi \lambda_{B} \omega^{2}(k)}{k^{2}}, \\
h_{\mathrm{MF}}(0)=-\frac{1}{2 \pi^{2}} \int_{0}^{\infty} d k \frac{4 \pi \lambda_{B} \omega^{2}(k) k^{2}}{k^{2}+4 \pi \lambda_{B} \rho \omega^{2}(k)} .
\end{gathered}
$$

We see that $h(r=0)<0$ regardless of the distribution $\omega(r)$. We conclude that soft interactions generated by charge spreading cannot lead to stacked configurations.

In Fig. 10, we show the lateral correlation function for the adsorbed counterions, $h_{\|}(r)$. Penetration reduces the degree

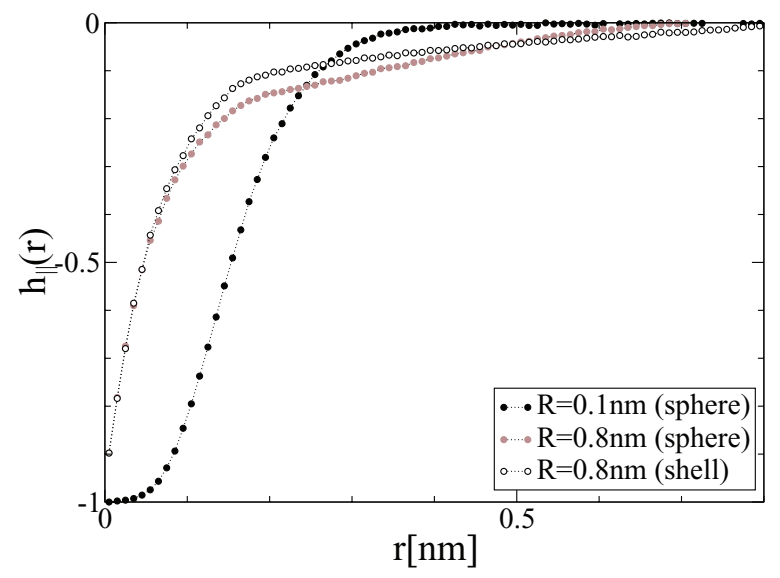

FIG. 10. Correlation function for counterions in the lateral plane adjacent to the wall. The layer thickness is $0.35 \mathrm{~nm}$. The remaining parameters are the same as in Fig. 7. The dashed lines guide the eye.

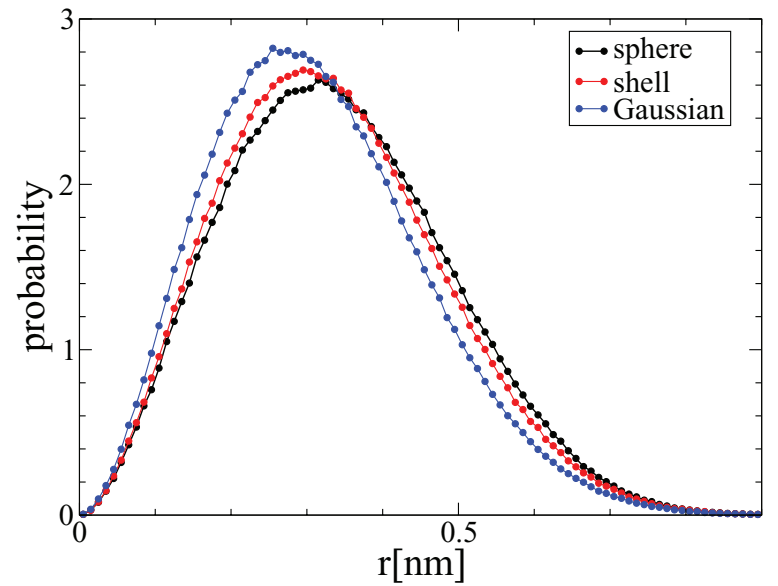

FIG. 11. The nearest neighbor separation distribution for different $\omega(r)$ functions. $R=0.8 \mathrm{~nm}$ and the same parameters as in Fig. 7 .

of electrostatic correlations between the ions, however, $h_{\|}(r)$ always remains negative and decreases monotonically all the way to $r=0$. The small difference between the shell and the sphere distributions indicates that the exact shape of the pair potential is unimportant.

In Fig. 11 we show the probability distribution of the nearest neighbor separation for various $\omega(r)$ functions. Differences are rather small and only quantitative. The Gaussian $\omega(r)$ shows preference for smaller interionic separation.

Finally, we explore the validity of the mean-field for multivalent symmetric ions, $Z>1$. We suppose that the meanfield should deteriorate quickly for $Z>1$ since the coupling constant $\Gamma \sim Z^{3}$. Figure 12 shows the counterion density profiles for $Z=2,3$. For $Z=2$ the mean-field is virtually exact and for $Z=3$ there are small deviations. This surprising agreement can be explained with the following. Increasing $Z$ naturally reduces the number of counterions required to neutralize the wall. On the other hand, a larger $Z$ generates stronger overcharging, so the density drop is partially compensated. The number of overlapping configurations is still large and the mean-field retains its validity. To confirm this conjecture, we check the $2 \mathrm{D}$ density of the counterions

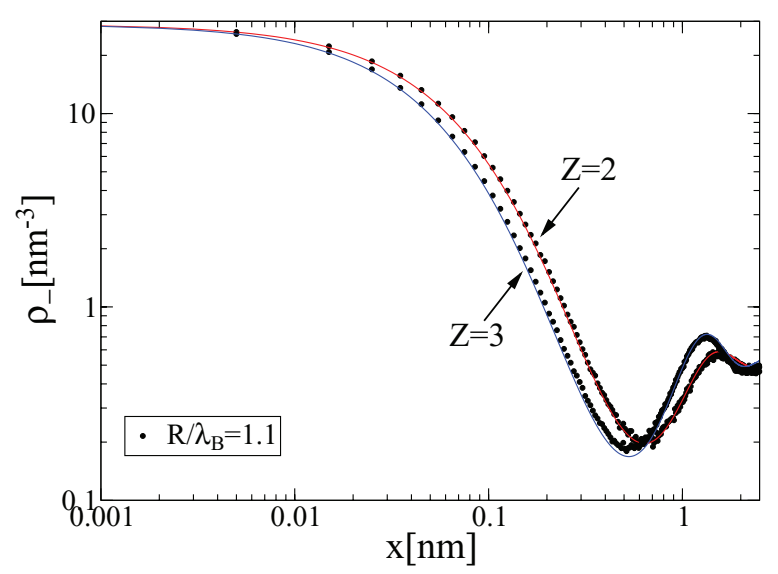

FIG. 12. Counterion distribution function for symmetric solution with ion charge $\pm e Z$. The remaining parameters are the same as in Fig. 7. The circles are the simulation data points and the lines are obtained from the FSPB. 
adsorbed on the charged wall. For $Z=3$, we find $R^{2} \rho_{2 D} \approx$ $1.2>1$. The overlapping configurations are, therefore, still significant.

\section{DISCUSSION}

For overcharging to occur, there must exist a mechanism that either reduces the free energy of counterions condensed on a charged surface (it becomes favorable for a counterion to leave its bulk environment) or increases the free energy of a bulk (it becomes less favorable for ions to stay in the bulk). It is the exchange between the two environments, the bulk and the double-layer, that leads to overcharging. We consider the environment of a charged surface with its adsorbed counterions and consider the conditions that lead to lowering of its energy. The surface charge of a plate is $\sigma_{c}$. We do not violate the condition of neutrality, thus the $2 \mathrm{D}$ density of adsorbed counterions is $\sigma_{c} / Z$. We focus on the energy of a single counterion. For sake of simplicity, we assume counterions and the charged wall occupy the same plane. An attraction to the wall yields a negative energy contribution,

$$
\begin{aligned}
\beta E_{\mathrm{wall}} & =\lim _{r_{c} \rightarrow \infty}\left[-2 \pi \int_{0}^{r_{c}} d r r \frac{Z \lambda_{B} \sigma_{c}}{r}\right] \\
& =\lim _{r_{c} \rightarrow \infty}\left[-2 \pi Z \lambda_{B} \sigma_{c} r_{c}\right],
\end{aligned}
$$

which is countered by the repulsive interactions with other counterions,

$$
\beta E_{\mathrm{int}}=\lim _{r_{c} \rightarrow \infty}\left[2 \pi \int_{0}^{r_{c}} d r \operatorname{rg}(r) \frac{Z \lambda_{B} \sigma_{c}}{r}\right],
$$

where $g(r)$ is the pair distribution function. For absence of correlations $g(r)=1$, and the attractive contribution is completely canceled out,

$$
\beta E_{\mathrm{int}}=\lim _{r_{c} \rightarrow \infty}\left[2 \pi Z \lambda_{B} \sigma_{c} r_{c}\right]
$$

But the cancellation will not be exact if correlations are present. Separating correlations from the pair distribution function, $g(r)=1+h(r)$, the uncanceled correlation energy is

$$
\beta E_{\text {corr }}=2 \pi \lambda_{B} \sigma_{c} \int_{0}^{\infty} d r h(r) .
$$

The relation between the correlation energy and the correlation function is quite clear. An ion will generate a negative correlation hole in its neighborhood, which will lead to negative energy contributions. We assume the following simple correlation hole, $h(r>\zeta)=0$ and $h(r<\zeta)=-1$, that is, by fixing a position of an ion, we introduce a circular hole in the density profile with the correlation length $\zeta$. The conservation of mass condition requires that an area of the hole is related to the density via $\sigma_{c} / Z=1 /\left(\pi \zeta^{2}\right)$, therefore, the correlation length is $\zeta=\sqrt{Z /\left(\pi \sigma_{c}\right)}$. This correlation function is the low temperature limit of the correlation hole obtained by construction from the linear mean-field treatment. ${ }^{41}$ Inserting this $h(r)$ into Eq. (37), we can approximate the correlation energy in the strong-coupling limit,

$$
\beta E_{\mathrm{corr}} \approx-2 \pi^{1 / 2} Z^{3 / 2} \lambda_{B} \sigma_{c}^{1 / 2}
$$

Recalling the definition of the coupling constant, $\Gamma$ $=2 \pi Z^{3} \lambda_{B}^{2} \sigma_{c}$, we get

$$
\beta E_{\mathrm{corr}} \approx-\sqrt{2 \Gamma}
$$

But for penetrable ions correlations are not required for reducing the electrostatic energy of counterions-the fact demonstrated by the validity of the mean-field theory. Consequently, we set $h(r)=0$. The reduction in electrostatic energy comes from a different source, from the fact that at overlapping separations, $r<2 R$, the electrostatic interactions are reduced on account of smearing procedure, which leads to the energy gain,

$$
\begin{aligned}
\beta E_{\text {overlap }} & \approx 2 \pi \int_{0}^{2 R} d r \sigma_{c} r\left[\beta U(r)-\frac{Z \lambda_{B}}{r}\right] \\
& =-C \pi Z \lambda_{B} \sigma_{c} R,
\end{aligned}
$$

where the constant $C$ depends on the pair potential $U(r)$, which, in turn, is determined by the distribution function $\omega(r)$. For the distributions considered in this work: $C_{\text {sphere }}=36 / 35$, $C_{\text {shell }}=4 / 3$, and $C_{\text {gauss }}=2.06$. Comparing Eq. (38) with Eq. (40), we see the different dependence of each mechanism on different parameters. The stabilization based on penetration has stronger dependence on the surface charge and the Bjerrum constant, on the other hand, its dependence on valency is weaker. In the strong-coupling limit, penetrable ions will exhibit correlated motions. If the correlation length is larger than the diameter of an ion, $\sqrt{Z /\left(\pi \sigma_{c}\right)}>2 R$, penetration may be neglected and the former mechanism comes to the fore. On the other hand, if $\sqrt{Z /\left(\pi \sigma_{c}\right)}<2 R$, we expect the two contributions to mix.

To recap, both mechanisms depend on eliminating the energy contributions coming from short separations between ions. For point ions in the strong-coupling limit, configurations with short separations are eliminated through correlated motion. The price is sacrifice in entropy, despite this, the total free energy is lowered. For penetrable ions the problem of high energy contributions at short separations does not exist to begin with. Due to smearing out procedure of an ion charge and the removal of the divergence from pair interactions, these contributions are taken out of the picture. Consequently, there is no entropy price to be paid, as all separations are explored "equally" and the mechanism is valid in the weak-coupling limit.

\section{CONCLUSION}

The present work studies the structure of a double-layer composed of ions whose central charge is smeared over a finite region in accordance with a weight function $\omega(r)$. The smearing-out procedure removes the divergence as $r \rightarrow 0$ from the pair interaction, allowing for interpenetration between the ions. The conditions under which penetration is favored are large temperature and high density. This regime is suitable for the mean-field treatment. Accordingly, we derive a modified Poisson-Boltzmann equation for spread-out charges (the FSPB equation). The FSPB equation predicts that for sufficiently large spreading radius $R$, overcharging takes 
place-the MC simulations verify this prediction. This suggests an alternative mechanism for charge reversal that is not related to correlations and the strong-coupling limit.

Using simulations and the mean-field approximation, we can exclude the ionic stacking as the underlying mechanism of overcharging. In fact, any soft potential obtained by charge spreading cannot lead to stacked configurations.

As a final consideration, we address the physical relevance of the smeared-out ion model. The spreading-out of the charge may capture the interactions between charges distributed along the polymer chains, but a more realistic representation would involve a non-electrostatic component produced by the self-avoidance of the polymer chains. Within the electrostatics framework, we have suggested a plausible distribution $\omega(r)$ composed of a charged shell and sphere, which generates an additional repulsion inside a penetrable core. In the end, however, a physically accurate pair potential requires corroboration with experimental analysis.

\section{ACKNOWLEDGMENTS}

D.F. is grateful to Nathan Berkovits for an invitation to and a short sojourn in the ICTP-SAIFR, where, prompted by the theoretical atmosphere of the institute, the formal aspects of the FSPB equation were laid down. This work was partially supported by the CNPq, FAPERGS, INCT-FCx, and by the U.S. AFOSR under the grant FA9550-12-1-0438.

${ }^{1}$ G. M. Torrie and J. P. Valleau, J. Chem. Phys. 73, 5807 (1980); E. GonzalesTovar, M. Lozada-Cassou, and D. Henderson, ibid. 83, 361 (1985); M. Lozada-Cassou, R. Saavedra-Barrera, and D. Henderson, ibid. 77, 5150 (1982).

${ }^{2}$ A. Yu. Grosberg, T. T. Nguyen, and B. I. Shklovskii, Rev. Mod. Phys. 74, 329 (2002); Y. Levin, Rep. Prog. Phys. 65, 1577 (2002); W. M. Gelbart, R. F. Bruinsma, P. A. Pincus, and V. A. Parsegian, Phys. Today 53, 38 (2000); F. J. Solis and M. O. de la Cruz, ibid. 54, 71 (2001).

${ }^{3}$ H. Greberg and R. Kjellander, J. Chem. Phys. 108, 2940 (1998).

${ }^{4}$ R. Messina, E. González-Tovar, M. Lozada-Cassou, and C. Holm, Europhys. Lett. 60, 383 (2002)

${ }^{5}$ G. I. Guerrero-Garcia, E. Gonzáles-Tovar, M. Chávez-Páez, and M. Lozada-Cassou, J. Chem. Phys. 132, 054903 (2010).

${ }^{6}$ D. Frydel and Y. Levin, J. Chem. Phys. 137, 164703 (2012).

${ }^{7}$ I. Rouzina and V. Bloomfield, J. Chem. Phys. 100, 9977 (1996).

${ }^{8}$ V. I. Perel and B. I. Shklovskii, Physica A 274, 446 (1999); B. I. Shklovskii, Phys. Rev. E 60, 5802 (1999); Phys. Rev. Lett. 82, 3268 (1999).
${ }^{9}$ S. Pianegonda, M. C. Barbosa, and Y. Levin, Europhys. Lett. 71, 831 (2005); A. Diehl and Y. Levin, J. Chem. Phys. 129, 124506 (2008); A. P. dos Santos, A. Diehl, and Y. Levin, ibid. 132, 104105 (2010).

${ }^{10}$ L. Šamaj and E. Trizac, Phys. Rev. Lett. 106, 078301 (2011).

${ }^{11}$ G. A. McConnell, A. P. Gast, J. S. Huang, and S. D. Smith, Phys. Rev. Lett. 71, 2102 (1993).

${ }^{12}$ C. N. Likos, A. Lang, M. Watzlawek, and H. Löwen, Phys. Rev. E 63, 031206 (2001).

${ }^{13}$ C. Marquest and T. A. Witten, J. Phys. (France) 50, 1267 (1989).

${ }^{14}$ C. N. Likos, M. Watzlawek, and H. Löwen, Phys. Rev. E 58, 3135 (1998).

${ }^{15}$ M. Schmidt, J. Phys.: Condens. Matter 11, 10163 (1999).

${ }^{16}$ Y. Rosenfeld, M. Schmidt, M. Watzlawek, and H. Löwen, Phys. Rev. E 62, 5006 (2000).

${ }^{17}$ D. Coslovich, J. P. Hansen, and G. Kahl, Soft Matter 7, 1690 (2011).

${ }^{18}$ D. Coslovich, J. P. Hansen, and G. Kahl, J. Chem. Phys. 134, 244514 (2011).

${ }^{19}$ A. Nikoubashman, J. P. Hansen, and G. Kahl, J. Chem. Phys. 137, 094905 (2012).

${ }^{20}$ M. E. Fisher and Y. Levin, Phys. Rev. Lett. 71, 3826 (1993).

${ }^{21}$ C. N. Likos, M. Schmidt, H. Löwen, M. Ballauff, D. Pötschke, and P. Lindner, Macromolecules 34, 2914 (2001).

${ }^{22}$ A. A. Louis, P. G. Bolhuis, J. P. Hansen, and E. J. Meijer, Phys. Rev. Lett. 85, 2522 (2000).

${ }^{23}$ J.-L. Barrat and F. Joanny, Adv. Chem. Phys. 94, 1 (1996).

${ }^{24}$ D. Gottwald, C. N. Likos, G. Kahl, and H. Löwen, Phys. Rev. Lett. 92, 068301 (2004).

${ }^{25}$ I. Borhukov, D. Andelman, and H. Orland, Phys. Rev. Lett. 79, 435 (1997).

${ }^{26}$ A. Abrashkin, D. Andelman, and H. Orland, Phys. Rev. Lett. 99, 077801 (2007).

${ }^{27}$ D. Frydel and M. Oettel, Phys. Chem. Chem. Phys. 13, 4109 (2011).

${ }^{28}$ D. Frydel, J. Phys. Chem. 134, 234704 (2011).

${ }^{29}$ M. M. Hatlo, R. van Roij, and L. Lue, Europhys. Lett. 97, 28010 (2012).

${ }^{30}$ Z.-G. Wang, Phys. Rev. E 81, 021501 (2010).

${ }^{31}$ P. Ewald, Ann. Phys. 369, 253 (1921).

${ }^{32}$ D. Frenkel and B. Smit, Understanding Molecular Simulation: From Algorithms to Applications, 2nd ed. (Academic Press, 2002); M. P. Allen and D. J. Tildesley, Computer Simulation of Liquids (Oxford University Press, 1989).

${ }^{33}$ Y. Rosenfeld, Phys. Rev. A 35, 938 (1987); J. Stein, D. Shalitin, and Y. Rosenfeld, ibid. 37, 4854 (1988).

${ }^{34}$ J.-P. Hansen and I. R. McDonald, Phys. Rev. A 23, 2041 (1981).

${ }^{35}$ A. A. Koulakov, M. M. Fogler, and B. I. Shklovskii, Phys. Rev. Lett. 76, 499 (1996).

${ }^{36}$ M. M. Fogler, A. A. Koulakov, and B. I. Shklovskii, Phys. Rev. B 54, 1853 (1996).

${ }^{37}$ A. R. Denton, Phys. Rev. E 67, 011804 (2003).

${ }^{38}$ A. G. Moreira and R. R. Netz, Europhys. Lett. 52, 705 (2000).

${ }^{39}$ F. H. Stillinger, J. Chem. Phys. 65, 3968 (1976); F. H. Stillinger and T. A. Weber, ibid. 68, 3837 (1978); F. H. Stillinger and D. K. Stillinger, Physica A 244, 358 (1997).

${ }^{40}$ A. Lang, C. N. Likos, M. Watzlawek, and H. Löwen, J. Phys.: Condens. Matter 12, 5087 (2000).

${ }^{41}$ S. Nordholm, Chem. Phys. Lett. 105, 302 (1984). 